\title{
Ordered Belief Fusion in Possibilistic Logic
}

\author{
Churn-Jung Liau \\ Institute of Information Science \\ Academia Sinica, Taipei, 115, Taiwan \\ liaucj@iis.sinica.edu.tw
}

\begin{abstract}
In this paper, we propose a logical framework for reasoning about uncertain belief fusion. The framework is a combination of multi-agent epistemic logic and possibilistic logic. We use graded epistemic operators to represent agents' uncertain beliefs, and the operators are interpreted in accordance with possibilistic semantics. Ordered fusion can resolve the inconsistency caused by direct fusion. We consider two strategies to merge uncertain beliefs. In the first strategy, called level cutting fusion, if inconsistency occurs at some level, then all beliefs at the lower levels are discarded simultaneously. In the second, called level skipping fusion, only the level at which the inconsistency occurs is skipped. We present the formal semantics and axiomatic systems for these two strategies.

Key Words: Belief fusion, database merging, epistemic logic, multiagent systems, possibilistic logic.
\end{abstract}

\section{Introduction}

The development of epistemic logic has been stimulated by the philosophical analysis of knowledge and belief [7]. This kind of logic has attracted the attention of researchers from diverse fields, such as artificial intelligence (AI), economics, linguistics, and theoretical computer science. Among them, AI researchers and computer scientists have developed some technically sophisticated formalisms and applied them to the analysis of distributed and multi-agent systems $[6,13]$.

The application of epistemic logic to AI and computer science emphasizes the interaction of agents, from which multi-agent epistemic logic has been developed. One representative example of such logic is proposed by Fagin et al. [6]. The term "knowledge" is used in a broad sense in [6] to cover cases of belief and information ${ }^{1}$. The most novel feature of their logic is its consideration of common knowledge and distributed knowledge among a group of agents. Distributed knowledge is that which can be deduced by pooling everyone's knowledge. In this paper, the distributed knowledge operator is also called the direct fusion operator. While it is essential that proper knowledge must be true, the belief of an agent may be wrong. Therefore, in general, there will be conflict

\footnotetext{
${ }^{1}$ More precisely, the logic for belief is called doxastic logic. However, here we use the three terms knowledge, belief, and information interchangeably, so epistemic logic is assumed to cover all these notions.
} 
between the beliefs to be merged. In this case, everything can be deduced from the distributed belief due to the notorious omniscience property of epistemic logic, so the merged result will be useless for further reasoning. To resolve the inconsistency of merged belief, ordered fusion operators are incorporated into multi-agent epistemic logic. This has resulted in the development of fusion logics $[3,4,8,9]$, in which the reliability ordering of agents is taken into account when their beliefs are merged.

While multi-agent epistemic logic does not consider the uncertainty of beliefs, a quantitative modal logic (QML) has been proposed for reasoning about such beliefs [10-12]. The direct fusion of uncertain beliefs is also considered in possibilistic logic, $\mathbf{P} \mathbf{L}_{n}^{\otimes}$, which extends QML with distributed belief operators [1]. The inconsistency problem in the direct fusion of beliefs also arises in the direct fusion of uncertain beliefs. Therefore, in this paper, we propose the ordered fusion of uncertain beliefs to resolve the problem.

\section{Review of Previous Approaches}

In this section, we review some logics for distributed belief fusion. For brevity, we only sketch the syntax and semantics of these logics, and omit their proof methods.

\subsection{Direct fusion in epistemic logic}

In [6], some variants of epistemic logic systems are presented. Using the naming convention in [2], the most basic system with distributed beliefs is called $\mathrm{K}_{n}^{D}$, with $n$ being the number of agents and $D$ denoting the distributed belief operators. In this system, logical omniscience is the only property imposed on agents' beliefs. Nevertheless, we further require that the belief of each individual agent should be consistent, even though the agents' collective beliefs may be in conflict. Thus, we actually use the $\operatorname{logic} \mathrm{KD}_{n}^{D}$ in [6], where an axiom $\mathrm{D}$ is used to guarantee the consistency of each agent's belief.

The alphabet of $\mathrm{KD}_{n}^{D}$ consists of the following symbols: a countable set $\Phi_{0}=$ $\{p, q, r, \ldots\}$ of atomic propositions; the propositional constants $\perp$ (falsum or falsity constant) and $T$ (verum or truth constant); the binary Boolean operator $\vee$ (or) and the unary Boolean operator $\neg$ (not); a set $A g=\{1,2, \ldots, n\}$ of agents; the modal operator-forming symbols "[" and "]"; and the left and right parentheses "(" and ")".

The set of well-formed formulas (wffs)is defined as the smallest set containing $\Phi_{0} \cup\{\perp, \top\}$ and is closed under Boolean operators and the following rule ${ }^{2}$ :

- if $\varphi$ is a wff, then $[G] \varphi$ is a wff for any nonempty $G \subseteq A g$.

The intuitive meaning of $[G] \varphi$ is "The group of agents $G$ has distributed belief $\varphi "$

${ }^{2}$ We change the syntactic notation of epistemic logic in [6] slightly. 
As usual, other classical Boolean connectives, such as $\wedge$ (and), $\supset$ (implication), and $\equiv$ (equivalence) can be defined as abbreviations. Also, we write $\langle G\rangle \varphi$ as an abbreviation of $\neg[G] \neg \varphi$. When $G$ is a singleton $\{i\}$, we write $[i] \varphi$ instead of $[\{i\}] \varphi$, so $[i] \varphi$ means that agent $i$ knows $\varphi$.

For the semantics, a possible world model for $\mathrm{KD}_{n}^{D}$ is a triple

$$
\left(W,\left(\mathcal{R}_{i}\right)_{1 \leq i \leq n}, V\right)
$$

where

- $W$ is a set of possible worlds,

- $\mathcal{R}_{i} \subseteq W \times W$ is a serial binary relation ${ }^{3}$ over $W$ for $1 \leq i \leq n$,

$-V: \Phi_{0} \rightarrow 2^{W}$ is a truth assignment mapping each atomic proposition to the set of worlds in which it is true.

From the binary relations, $\mathcal{R}_{i}$ 's, we can define a derived relation, $\mathcal{R}_{G}$, for each nonempty $G \subseteq A g$ :

$$
\mathcal{R}_{G}=\cap_{i \in G} \mathcal{R}_{i} .
$$

Note that the seriality of $\mathcal{R}_{i}$ guarantees the consistency of each agent's belief state. However, $\mathcal{R}_{G}$ may be not serial.

Informally, $\mathcal{R}_{i}(w)$ is the set of worlds that agent $i$ considers possible under $w$ according to his belief, so $\mathcal{R}_{G}(w)$ is the set of worlds that are considered possible under $w$ according to the direct fusion of agents' beliefs. This informal intuition is reflected in the definition of the satisfaction relation. Let $M=\left(W,\left(\mathcal{R}_{i}\right)_{1 \leq i \leq n}, V\right)$ be a model and $\mathcal{L}$ be the set of wffs for $\mathrm{KD}_{n}^{D}$. The satisfaction relation $\models{ }_{M} \subseteq$ $W \times \mathcal{L}$ is then defined by the following inductive rules (we use the infix notation for the relation and omit the subscript $M$ for convenience):

1. $w \models p$ iff $w \in V(p)$, for each $p \in \Phi_{0}$,

2. $w \not \models \perp$ and $w \models \top$,

3. $w \models \neg \varphi$ iff $w \not \models \varphi$,

4. $w \models \varphi \vee \psi$ iff $w \models \varphi$ or $w \models \psi$,

5. $w \models[G] \varphi$ iff for all $u \in \mathcal{R}_{G}(w), u \models \varphi$.

\subsection{Ordered fusion in epistemic logic}

To encode the degrees of reliability of $n$ agents, we use ordering relations over any subset of $\{1, \ldots, n\}$. Let $\mathcal{T} \mathcal{O}_{n}$ denote the set of all possible strict total orders over any non-empty subset of $\{1, \ldots, n\}$; then we can associate a unique syntactic notation with each total order in $\mathcal{T} \mathcal{O}_{n}$. Let $X=\left\{i_{1}, i_{2}, \ldots, i_{m}\right\}$ be a non-empty subset of $\{1, \ldots, n\}$ and $>$ be a strict total order such that $i_{j}>i_{k}$ iff $j<k$ for all $1 \leq, j, k \leq m$; then the syntactic notation for $(X,>)$ is the string

$$
i_{1}>i_{2}>\cdots>i_{m}
$$

\footnotetext{
${ }^{3}$ A binary relation $\mathcal{R}$ is serial if $\forall w \exists u$. $\mathcal{R}(w, u)$.
} 
In this paper, the capital letter $O$ is used to denote meta-variables ranging over such notations. Let $O$ be the string $i_{1}>i_{2}>\cdots>i_{m}$; then the set $\left\{i_{1}, i_{2}, \ldots, i_{m}\right\}$ is called the domain of $O$ and denoted by $\delta(O)$. In this case, $O>i_{m+1}$ denotes $i_{1}>i_{2}>\cdots>i_{m}>i_{m+1}$ if $i_{m+1} \notin \delta(O)$. As the syntactic notation is unique for each total order, we can also identify the notation with the total order itself, so we can write $O \in \mathcal{T} \mathcal{O}_{n}$. Furthermore, the upper-case Greek letter $\Omega$ is used to denote meta-variables ranging over nonempty subsets of $\mathcal{T} \mathcal{O}_{n}$.

In [9], two logics for ordered distributed belief fusion are proposed. The first, $\mathrm{DBF}_{n}^{c}$, is based on a level cutting strategy. The set of $\mathrm{DBF}_{n}^{c}$ wffs is defined by the rules for $\mathrm{KD}_{n}^{D}$ and the following rule:

- if $\varphi$ is a wff, then $[O] \varphi$ is a wff for any $O \in \mathcal{T} \mathcal{O}_{n}$.

Intuitively, $[O] \varphi$ means that $\varphi$ is derivable from the merged beliefs of agents in $\delta(O)$ according to the specific order of $O$.

For the semantics, a $\mathrm{DBF}_{n}^{c}$ model is a possible world model $\left(W,\left(\mathcal{R}_{i}\right)_{1 \leq i \leq n}, V\right)$ for $\mathrm{KD}_{n}^{D}$. For each $O \in \mathcal{T} \mathcal{O}_{n}$, a derived relation, $\mathcal{R}_{O}^{c}$, is defined inductively as follows:

$$
\mathcal{R}_{O>i}^{c}(w)= \begin{cases}\mathcal{R}_{O}^{c}(w) & \text { if } \bigcap_{j \in \delta(O>i)} \mathcal{R}_{j}(w)=\emptyset, \\ \mathcal{R}_{O}^{c}(w) \cap \mathcal{R}_{i}(w) \text { otherwise, }\end{cases}
$$

for any $w \in W$. The superscript $c$ denotes level cutting fusion and can usually be omitted when the context is clear. The following satisfaction condition is then added to those of epistemic logic:

$$
w=[O] \varphi \text { iff for all } u \in \mathcal{R}_{O}(w), u \models \varphi .
$$

Let $O=i_{1}>i_{2}>\cdots>i_{m}$. Also, define $G_{j}=\left\{i_{1}, i_{2} \ldots, i_{j}\right\}$ for $1 \leq j \leq m$ and assume $k$ is the largest $j$ such that $\bigcap_{i \in G_{j}} \mathcal{R}_{i}(w) \neq \emptyset$; then we have

$$
\mathcal{R}_{O}(w)=\bigcap_{i \in G_{k}} \mathcal{R}_{i}(w)
$$

In other words, beliefs from agents below level $k$ are completely discarded from the merged result. Our rationale is that if a belief in level $k+1$ is unacceptable, then any belief in a less reliable level is also unacceptable.

The second logic, $\mathrm{DBF}_{n}^{s}$, is based on a level skipping strategy, which only skips the agent causing the inconsistency and continues to consider the next level. This strategy corresponds to the suspicious attitude of multi-source reasoning [3], and has also been used in belief revision by Nebel [14]. The set of $\mathrm{DBF}_{n}^{s}$ is the smallest set containing $\Phi_{0} \cup\{\perp, \top\}$, and is closed under Boolean operators and the following rule:

- if $\varphi$ is a wff, so is $[\Omega] \varphi$ for any nonempty $\Omega \subseteq \mathcal{T} \mathcal{O}_{n}$.

When $\Omega$ is a singleton $\{O\}$, we write $[O] \varphi$ instead of $[\{O\}] \varphi$. If $\Omega=\left\{O_{1}, \ldots, O_{m}\right\}$ such that $\left|\delta\left(O_{i}\right)\right|=1$ for all $1 \leq i \leq m$, then $[\Omega]$ is the distributed belief operator among ordinary agents. Therefore, the language is more general than that of $\mathrm{DBF}_{n}^{c}$. 
For the semantics, a $\mathrm{DBF}_{n}^{s}$ model is still a possible world model $\left(W,\left(\mathcal{R}_{i}\right)_{1 \leq i \leq n}, V\right)$ for $\mathrm{KD}_{n}^{D}$. Therefore, we can define $\mathcal{R}_{O}^{s}$ inductively as follows:

$$
\mathcal{R}_{O>i}^{s}(w)= \begin{cases}\mathcal{R}_{O}^{s}(w) & \text { if } \mathcal{R}_{O}^{s}(w) \cap \mathcal{R}_{i}(w)=\emptyset, \\ \mathcal{R}_{O}^{s}(w) \cap \mathcal{R}_{i}(w) \text { otherwise, }\end{cases}
$$

for any $w \in W$. As in the case of $\mathcal{R}_{O}^{c}$, the superscript $s$ denotes the level skipping strategy and can be omitted when the context is clear. We further define

$$
\mathcal{R}_{\Omega}=\bigcap_{O \in \Omega} \mathcal{R}_{O}
$$

Then, the following clause is used to define the satisfaction of modal formulas in $\mathrm{DBF}_{n}^{s}$.

$-w \models[\Omega] \varphi$ iff for all $u \in \mathcal{R}_{\Omega}(w), u \models \varphi$.

\subsection{Direct fusion in possibilistic logic}

In [1], a logic $\mathbf{P} \mathbf{L}_{n}^{\otimes}$ is proposed for reasoning about distributed belief fusion with a continuous T-norm $\otimes^{4}$. The set of $\mathbf{P} \mathbf{L}_{n}^{\otimes}$ wffs is the smallest set containing $\Phi_{0} \cup\{\perp, \top\}$, and is closed under Boolean operators and the following rule:

- if $\varphi$ is a wff, so are $B_{a}^{i} \varphi$ and $D_{a} \varphi$ for any $1 \leq i \leq n$ and rational number $a \in[0,1]$.

The intuitive meaning of $B_{a}^{i} \varphi$ is that agent $i$ believes $\varphi$ with strength (at least) $a$, and the modal operator, $D_{a}$, represents the distributed beliefs of all agents with strength (at least) $a$.

Formally, the semantics of $\mathbf{P L}_{n}^{\otimes}$ is based on possibility theory [15]. A $\Pi_{n}^{\otimes}$ structure is a tuple $\left(W,\left(\pi_{i}\right)_{0 \leq i \leq n}, V\right)$ such that $W$ is a set of possible worlds; each $\pi_{i}$ maps each world $w$ to a possibility distribution $\pi_{i, w}: W \rightarrow[0,1]$ over $W ; V$ maps elements in $\Phi_{0}$ to subsets of $W$; and, for any $w \in W$,

$$
\pi_{0, w} \leq \bigotimes_{i=1}^{n} \pi_{i, w}
$$

In possibility theory, each possibility distribution $\pi$ can derive the associated possibility measure $\Pi: 2^{W} \rightarrow[0,1]$ and necessity measure $N: 2^{W} \rightarrow[0,1]$ as

$$
\begin{gathered}
\Pi(X)=\sup _{x \in X} \pi(x) \\
N(X)=1-\sup _{x \notin X} \pi(x) .
\end{gathered}
$$

Then, the satisfaction relation $\models$ for $\Pi_{n}^{\otimes}$-structures are defined as

$-w \models B_{a}^{i} \varphi$ iff $N_{i, w}(|\varphi|) \geq a$,

$-w \models D_{a} \varphi$ iff $N_{0, w}(|\varphi|) \geq a$,

where $|\varphi|=\{x \in W \mid x \models \varphi\}$ is the truth set of $\varphi$ in the model, and $N_{i, w}$ is the necessity measure associated to $\pi_{i, w}$ for $0 \leq i \leq n$ and $w \in W$.

${ }^{4} \mathrm{~A}$ T-norm is any binary operation on $[0,1]$ that is commutative, associative, and non-decreasing in each argument, and has 1 as its unit. 


\section{Ordered Fusion in Possibilistic Logic}

To resolve the inconsistency problem in $\mathbf{P L}_{n}^{\otimes}$, we combine $\mathrm{DBF}_{n}^{c}$ (resp. $\mathrm{DBF}_{n}^{s}$ ) with $\mathbf{P L}_{n}^{\otimes}$. Since possibilistic logic is inconsistency-tolerant [5], we introduce a parameter, $\epsilon$, to denote the degree of inconsistency tolerance. Recall that a possibility distribution $\pi: X \rightarrow[0,1]$ is normalized if $\Pi(X)=\sup _{x \in X} \pi(x)=1$. A normalized possibility distribution represents a consistent belief state. If $\pi$ is not normalized, i.e., $\sup _{x \in X} \pi(x)<1, \pi$ represents a partially inconsistent belief state. $1-\sup _{x \in X} \pi(x)$ is called the inconsistency degree of $\pi$, and denoted by $\iota(\pi)$.

\subsection{Level cutting fusion in possibilistic logic}

In this subsection, we present a logic for reasoning about possibilistic belief fusion based on a level cutting strategy. The logic is called $\mathrm{CFPL}_{n}^{\otimes, \epsilon}$, where $\epsilon$ is the inconsistency tolerance degree of the logic. The set of $\mathrm{CFPL}_{n}^{\otimes, \epsilon}$ wffs is defined as the smallest set containing $\Phi_{0} \cup\{\perp, \top\}$, and is closed under Boolean operators and the following rule:

- if $\varphi$ is a wff, then $[G]_{a} \varphi,[O]_{a} \varphi,[G]_{a}^{+} \varphi$, and $[O]_{a}^{+} \varphi$ are wffs for any nonempty $G \subseteq A g$, any $O \in \mathcal{T} \mathcal{O}_{n}$, and any rational number $a \in[0,1]$.

As in epistemic logic, we use $[i]$ instead of $[\{i\}]$ when $\{i\}$ is a singleton. The intuitive meanings of $[i]_{a} \varphi$ and $[A g]_{a} \varphi$ are respectively the same as those of $B_{a}^{i} \varphi$ and $D_{a} \varphi$ in $\mathbf{P} \mathbf{L}_{n}^{\otimes}$. However, we not only consider a single agent and the set of all agents, but also any nonempty subset of agents. $[G]_{a}^{+} \varphi$ is similar to $[G]_{a} \varphi$, except that the former means the strength of belief is greater than $a$. Additionally, we have modal operators corresponding to the ordered fusion of uncertain beliefs. $[O]_{a} \varphi$ (resp. $[O]_{a}^{+} \varphi$ ) means that an agent merging distributed beliefs in accordance with the ordering $O$ will believe $\varphi$ with a strength of at least (resp. more than) $a$.

For the semantics, a $\mathrm{CFPL}_{n}^{\otimes, \epsilon}$-model is a tuple $M=\left(W,\left(\pi_{i}\right)_{1 \leq i \leq n}, V\right)$ such that $W$ is a set of possible worlds; each $\pi_{i}$ maps each world $w$ to a possibility distribution $\pi_{i, w}: W \rightarrow[0,1]$ over $W$ such that $\iota\left(\pi_{i, w}\right) \leq \epsilon$; and $V$ maps elements in $\Phi_{0}$ to subsets of $W$. Note that we require the inconsistency degree of the belief state of each single agent to be no more than $\epsilon$. This is the inconsistency tolerance degree of the logic. Any belief with inconsistency beyond this degree must be discarded. Let us now define derived possibility distributions $\pi_{G, w}$ and $\pi_{O, w}$ from $\left\{\pi_{i} \mid 1 \leq i \leq n\right\}$ for each nonempty subset $G \subseteq A g, O \in \mathcal{T} \mathcal{O}_{n}$, and $w \in W$ as follows:

$$
\begin{gathered}
\pi_{G, w}=\bigotimes_{i \in G} \pi_{i, w} \\
\pi_{O>i, w}= \begin{cases}\pi_{O, w} & \text { if } \iota\left(\bigotimes_{j \in \delta(O>i)} \pi_{j, w}\right)>\epsilon, \\
\pi_{O, w} \otimes \pi_{i, w} & \text { otherwise, }\end{cases}
\end{gathered}
$$

Then, the satisfaction relation $\models$ for the $\mathrm{CFPL}_{n}^{\otimes, \epsilon}$-model is defined as 


$$
\begin{aligned}
& -w \models[G]_{a} \varphi \text { iff } N_{G, w}(|\varphi|) \geq a, \\
& -w \models[G]_{a}^{+} \varphi \text { iff } N_{G, w}(|\varphi|)>a, \\
& -w \models[O]_{a} \varphi \text { iff } N_{O, w}(|\varphi|) \geq a, \\
& -w \models[O]_{a}^{+} \varphi \text { iff } N_{O, w}(|\varphi|)>a,
\end{aligned}
$$

where $|\varphi|$ is the truth set of $\varphi$ in the model, and $N_{G, w}$ (resp. $N_{O, w}$ ) is the necessity measure associated with $\pi_{G, w}\left(\right.$ resp. $\left.\pi_{O, w}\right)$ for $G \subseteq A g$ (resp. $\left.O \in \mathcal{T} \mathcal{O}_{n}\right)$ and $w \in W$.

A set of wffs $\Sigma$ is satisfied in a world $w$, written as $w \models \Sigma$, if $w \models \varphi$ for all $\varphi \in \Sigma$. We write $\Sigma \models_{M} \varphi$ if for each possible world $w$ in $M, w \models \Sigma$ implies $w \models \varphi$, and $\Sigma=_{\mathrm{CFPL}_{n}^{\otimes, \epsilon}} \varphi$ if $\Sigma=_{M} \varphi$ for each $\mathrm{CFPL}_{n}^{\otimes, \epsilon}$-model $M$. A wff $\varphi$ is valid in $M$ if $\emptyset=_{M} \varphi$. $\Sigma$ can be omitted when it is empty. Thus, $\models_{M} \varphi$ and $=_{\mathrm{CFPL}_{n}^{\otimes, \epsilon}} \varphi$ are the abbreviations of $\emptyset \models_{M} \varphi$ and $\emptyset \models_{\mathrm{CFPL}_{n}^{\otimes, \epsilon}} \varphi$ respectively. The subscript is also usually omitted if it is clear from the context.

An axiomatic system for $\mathrm{CFPL}_{n}^{\otimes, \epsilon}$ is presented in Figure 1. The system was developed by generalizing $\mathrm{KD}_{n}^{D}$ to QML [10-12]. However, the consistency of each individual agent's belief is replaced by $(1-\epsilon)$-consistency, which means that the inconsistency degree of each agent's belief state is at most $\epsilon$. Axiom G2 enforces this requirement. The axioms governing modal operators $[O]_{a}$ and $[O]_{a}^{+}$ are generalized from those of $\mathrm{DBF}_{n}^{c}$. Also, the symbol $\oplus$ in axiom G3 denotes a T-conorm corresponding to $\otimes$, which is defined by $a \oplus b=1-(1-a) \otimes(1-b)$.

A wff $\varphi$ is derivable from the system $\mathrm{CFPL}_{n}^{\otimes, \epsilon}$, or simply, $\varphi$ is a theorem of $\mathrm{CFPL}_{n}^{\otimes, \epsilon}$, if there is a finite sequence $\varphi_{1}, \ldots, \varphi_{m}$ such that $\varphi=\varphi_{m}$ and every $\varphi_{i}$ is an instance of an axiom schema, or obtained from earlier $\varphi_{j}$ 's by the application of an inference rule. It is written as $\vdash_{\mathrm{CFPL}_{n}^{\otimes, \epsilon}} \varphi$ if $\varphi$ is a theorem of $\mathrm{CFPL}_{n}^{\otimes, \epsilon}$. Let $\Sigma \cup\{\varphi\}$ be a subset of wffs, then $\varphi$ is derivable from $\Sigma$ in the system $\mathrm{CFPL}_{n}^{\otimes, \epsilon}$, written as $\Sigma \vdash_{\mathrm{CFPL}_{n}^{\otimes, \epsilon}} \varphi$, if there is a finite subset $\Sigma^{\prime}$ of $\Sigma$ such that $\vdash_{\mathrm{CFPL}_{n}^{\otimes}, \epsilon} \bigwedge \Sigma^{\prime} \supset \varphi$. We drop the subscript when no confusion occurs.

We now have the soundness and completeness results for the system $\mathrm{CFPL}_{n}^{\otimes, \epsilon}$.

Theorem 1 For any wff of $C F P L_{n}^{\otimes, \epsilon}, \models \varphi$ iff $\vdash \varphi$.

\subsection{Level skipping fusion in possibilistic logic}

In this subsection, we present a logic for reasoning about possibilistic belief fusion based on a level skipping strategy. The logic is called $\mathrm{SFPL}_{n}^{\otimes, \epsilon}$, where $\epsilon$ is the inconsistency tolerance degree of the logic. The set of SFPL ${ }_{n}^{\otimes, \epsilon}$ wffs is defined as the smallest set containing $\Phi_{0} \cup\{\perp, T\}$, and is closed under Boolean operators and the following rule:

- if $\varphi$ is a wff, then $[\Omega]_{a} \varphi$ and $[\Omega]_{a}^{+} \varphi$ are wffs for any nonempty $\Omega \subseteq \mathcal{T} \mathcal{O}_{n}$ and any rational number $a \in[0,1]$.

Semantically, an $\mathrm{SFPL}_{n}^{\otimes, \epsilon}$-structure is the same as a $\mathrm{CFPL}_{n}^{\otimes, \epsilon}{ }_{\text {-structure. }}$ However, we redefine $\pi_{O, w}$ for each $O \in \mathcal{T} \mathcal{O}_{n}$ and $w \in W$ as follows:

$$
\pi_{O>i, w}= \begin{cases}\pi_{O, w} & \text { if } \iota\left(\pi_{O, w} \otimes \pi_{i, w}\right)>\epsilon, \\ \pi_{O, w} \otimes \pi_{i, w} & \text { otherwise. }\end{cases}
$$


- Axioms:

1. P: all tautologies of propositional calculus

2. Bookkeeping ( $\square$ denotes either $[G]$ or $[O]$ ):
(a) $\square_{c} \varphi \supset \square_{d}^{+} \varphi$ if $c>d$
(b) $\square_{c}^{+} \varphi \supset \square_{c} \varphi$
(c) $\square_{0} \varphi$
(d) $\neg \square \square_{1}^{+} \varphi$

3. G1:

(a) $\left([G]_{a} \varphi \wedge[G]_{a}(\varphi \supset \psi)\right) \supset[G]_{a} \psi$

(b) $\left([G]_{a}^{+} \varphi \wedge[G]_{a}^{+}(\varphi \supset \psi)\right) \supset[G]_{a}^{+} \psi$

4. $\mathrm{G} 2: \neg[i]_{\epsilon}^{+} \perp$

5. G3: if $G_{1} \cap G_{2}=\emptyset$, then

(a) $\left(\left[G_{1}\right]_{a} \varphi \wedge\left[G_{2}\right]_{b} \varphi\right) \supset\left[G_{1} \cup G_{2}\right]_{a \oplus b} \varphi$

(b) $\left(\left[G_{1}\right]_{a}^{+} \varphi \wedge\left[G_{2}\right]_{b}^{+} \varphi\right) \supset\left[G_{1} \cup G_{2}\right]_{a \oplus b}^{+} \varphi$

6. $\mathrm{O} 1$
(a) $\neg[\delta(O>i)]_{\epsilon}^{+} \perp \supset\left([O>i]_{a} \varphi \equiv[\delta(O>i)]_{a} \varphi\right)$
(b) $\neg[\delta(O>i)]_{\epsilon}^{+} \perp \supset\left([O>i]_{a}^{+} \varphi \equiv[\delta(O>i)]_{a}^{+} \varphi\right)$

7. $\mathrm{O} 2$ :

(a) $[\delta(O>i)]_{\epsilon}^{+} \perp \supset\left([O>i]_{a} \varphi \equiv[O]_{a} \varphi\right)$

(b) $[\delta(O>i)]_{\epsilon}^{+} \perp \supset\left([O>i]_{a}^{+} \varphi \equiv[O]_{a}^{+} \varphi\right)$

- Rules of Inference:

1. R1 (Modus ponens, MP):

$$
\frac{\varphi \varphi \supset \psi}{\psi}
$$

2. R2 (Generalization, Gen):

$$
\frac{\varphi}{[G]_{1} \varphi}
$$

Fig. 1. The axiomatic system for $\mathrm{CFPL}_{n}^{\otimes, \epsilon}$

Furthermore, we also define $\pi_{\Omega, w}$ for each $\Omega \subseteq \mathcal{T O}_{n}$ and $w \in W$ as

$$
\pi_{\Omega, w}=\bigotimes_{O \in \Omega} \pi_{O, w}
$$

Then, the satisfaction relation $\models$ for the $\mathrm{SFPL}_{n}^{\otimes, \epsilon}$-model is defined as

$-w \models[\Omega]_{a} \varphi$ iff $N_{\Omega, w}(|\varphi|) \geq a$,

$-w \models[\Omega]_{a}^{+} \varphi$ iff $N_{\Omega, w}(|\varphi|)>a$,

where $|\varphi|$ is the truth set of $\varphi$ in the model, and $N_{\Omega, w}$ is the necessity measure associated with $\pi_{\Omega, w}$. The definition of the validity and consequence relation is the same as above.

An axiomatic system can be also developed for $\mathrm{SFPL}_{n}^{\otimes, \epsilon}$ by generalizing the corresponding axioms in $\mathrm{DBF}_{n}^{s}$, as shown in Figure 2. 
- Axioms:

1. P: all tautologies of propositional calculus

2. Bookkeeping:
(a) $[\Omega]_{c} \varphi \supset[\Omega]_{d}^{+} \varphi$ if $c>d$
(b) $[\Omega]_{c}^{+} \varphi \supset[\Omega]_{c} \varphi$
(c) $[\Omega]_{0} \varphi$
(d) $\neg[\Omega]_{1}^{+} \varphi$

3. V1:
(a) $\left([\Omega]_{a} \varphi \wedge[\Omega]_{a}(\varphi \supset \psi)\right) \supset[\Omega]_{a} \psi$
(b) $\left([\Omega]_{a}^{+} \varphi \wedge[\Omega]_{a}^{+}(\varphi \supset \psi)\right) \supset[\Omega]_{a}^{+} \psi$

4. V2: $\neg[i]_{\epsilon}^{+} \perp$

5. V3: if $\Omega_{1} \cap \Omega_{2}=\emptyset$, then

(a) $\left(\left[\Omega_{1}\right]_{a} \varphi \wedge\left[\Omega_{2}\right]_{b} \varphi\right) \supset\left[\Omega_{1} \cup \Omega_{2}\right]_{a \oplus b} \varphi$

(b) $\left(\left[\Omega_{1}\right]_{a}^{+} \varphi \wedge\left[\Omega_{2}\right]_{b}^{+} \varphi\right) \supset\left[\Omega_{1} \cup \Omega_{2}\right]_{a \oplus b}^{+} \varphi$

6. $\mathrm{O} 1$
(a) $\neg[\{O, i\}]_{\epsilon}^{+} \perp \supset\left([\Omega \cup\{O>i\}]_{a} \varphi \equiv[\Omega \cup\{O, i\}]_{a} \varphi\right)$
(b) $\neg[\{O, i\}]_{\epsilon}^{+} \perp \supset\left([\Omega \cup\{O>i\}]_{a}^{+} \varphi \equiv[\Omega \cup\{O, i\}]_{a}^{+} \varphi\right)$

7. O2:

(a) $[\{O, i\}]_{\epsilon}^{+} \perp \supset\left([\Omega \cup\{O>i\}]_{a} \varphi \equiv[\Omega \cup\{O\}]_{a} \varphi\right)$

(b) $[\{O, i\}]_{\epsilon}^{+} \perp \supset\left([\Omega \cup\{O>i\}]_{a}^{+} \varphi \equiv[\Omega \cup\{O\}]_{a}^{+} \varphi\right)$

- Rules of Inference:

1. R1 (Modus ponens, MP):

$$
\frac{\varphi \varphi \supset \psi}{\psi}
$$

2. R2 (Generalization, Gen):

$$
\frac{\varphi}{[\Omega]_{1} \varphi}
$$

Fig. 2. The axiomatic system for $\mathrm{SFPL}_{n}^{\otimes, \epsilon}$

The definition of derivability and theoremhood in the $\mathrm{SFPL}_{n}^{\otimes, \epsilon}$ system is the same as above. We now have the soundness and completeness theorem for $\mathrm{SFPL}_{n}^{\otimes, \epsilon}$.

Theorem 2 For any wff of $S F P L_{n}^{\otimes, \epsilon}, \models \varphi$ iff $\vdash \varphi$.

\section{Concluding Remarks}

In this paper, we present two logics for reasoning about ordered possibilistic belief fusion. Direct fusion and ordered fusion in epistemic logic, as well as direct fusion in possibilistic logic have been proposed in the previous literature. Therefore, the results in this paper fill a gap in the previous work. We believe that the logics, which are summarized in Table 1, are applicable to reasoning in multi-agent systems. 
Table 1. Logics for belief fusion

\begin{tabular}{|c|c|c|}
\hline & without uncertainty & with uncertainty \\
\hline direct fusion & $\mathrm{KD}_{n}^{D}$ & $\mathrm{DBF}_{n}^{c} / \mathrm{DBF}_{n}^{s}$ \\
\hline ordered fusion & $\mathbf{P L}_{n}^{\otimes}$ & $\mathrm{CFPL}_{n}^{\otimes, \epsilon} / \mathrm{SFPL}_{n}^{\otimes, \epsilon}$ \\
\hline
\end{tabular}

\section{References}

1. Boldrin L., Saffiotti A.: A modal logic for merging partial belief of multiple reasoners. Journal of Logic and Computation 9 (1999) 81-103

2. B.F. Chellas. Modal Logic: An Introduction. Cambridge University Press, 1980.

3. L. Cholvy. A logical approach to multi-souces reasoning. In M. Masuch and L. Pólos, editors, Knowledge Representation and Reasoning under Uncertainty, LNCS 808, pages 183-196. Springer-Verlag, 1994.

4. L. Cholvy. Reasoning about data provided by federated deductive databases. Journal of Intelligent Information Systems, 10:49-80, 1998.

5. D. Dubois, J. Lang, and H. Prade. Possibilistic logic. In D.M. Gabbay, C.J. Hogger, and J.A. Robinson, editors, Handbook of Logic in Artificial Intelligence and Logic Programming, Vol 3 : Nonmonotonic Reasoning and Uncertain Reasoning, pages 439-513. Clarendon Press - Oxford, 1994.

6. R. Fagin, J.Y. Halpern, Y. Moses, and M.Y. Vardi. Reasoning about Knowledge. MIT Press, 1996.

7. J. Hintikka. Knowledge and Belief. Cornell University Press, 1962.

8. C. J. Liau. A conservative approach to distributed belief fusion. In Proc. of the Third International Conference on Information Fusion, pages MoD4-1, 2000.

9. C.J. Liau. A modal logic framework for multi-agent belief fusion. ACM Transactions on Computational Logic, 6(1):124-174, 2005.

10. C.J. Liau and I.P. Lin. Quantitative modal logic and possibilistic reasoning. In B. Neumann, editor, Proceedings of the 10th ECAI, pages 43-47. John Wiley \& Sons. Ltd, 1992.

11. C.J. Liau and I.P. Lin. Proof methods for reasoning about possibility and necessity. International Journal of Approximate Reasoning, 9(4):327-364, 1993.

12. C.J. Liau and I.P. Lin. Possibilistic reasoning - a mini-survey and uniform semantics. Artificial Intelligence, 88:163-193, 1996.

13. J.-J. Ch. Meyer and W. van der Hoek. Epistemic Logic for AI and Computer Science. Cambridge University Press, 1995.

14. B. Nebel. Base revision operator and schemes: semantics representation and complexity. In Proceedings of the 11th European Conference on Artificial Intelligence, pages 341-345. John Wiley \& Sons, 1994.

15. L.A. Zadeh. Fuzzy sets as a basis for a theory of possibility. Fuzzy Sets and Systems, 1(1):3-28, 1978. 